\title{
Short-term outcomes of minimally invasive retromuscular ventral hernia repair using an enhanced view totally extraperitoneal (eTEP) approach: systematic review and meta-analysis
}

\author{
D. Aliseda ${ }^{1}$ (D) $\cdot$ C. Sanchez-Justicia ${ }^{1,2} \cdot$ G. Zozaya ${ }^{1,2} \cdot$ J. Lujan $^{1} \cdot$ A. Almeida ${ }^{1} \cdot$ N. Blanco ${ }^{1} \cdot$ P. Martí-Cruchaga $^{1,2}$. \\ F. Rotellar ${ }^{1,2}$
}

Received: 24 November 2021 / Accepted: 27 December 2021 / Published online: 19 January 2022

(c) The Author(s) 2022

\begin{abstract}
Background The enhanced view totally extraperitoneal (eTEP) approach is becoming increasingly more widely accepted as a promising technique in the treatment of ventral hernia. However, evidence is still lacking regarding the perioperative, postoperative and long-term outcomes of this technique. The aim of this meta-analysis is to summarize the current available evidence regarding the perioperative and short-term outcomes of ventral hernia repair using eTEP.

Study design A systematic search was performed of PubMed, EMBASE, Cochrane Library and Web of Science electronic databases to identify studies on the laparoscopic or robotic-enhanced view totally extraperitoneal (eTEP) approach for the treatment of ventral hernia. A pooled meta-analysis was performed. The primary end point was focused on short-term outcomes regarding perioperative characteristics and postoperative parameters.

Results A total of 13 studies were identified involving 918 patients. Minimally invasive eTEP resulted in a rate of surgical site infection of $0 \%$ [95\% CI 0.0-1.0\%], a rate of seroma of 5\% [95\% CI 2.0-8.0\%] and a rate of major complications (Clavien-Dindo III-IV) of $1 \%$ [95\% CI 0.0-3.0\%]. The rate of intraoperative complications was 2\% [95\% CI 0.0-4.0\%] with a conversion rate of $1.0 \%$ [95\% CI 0.0-3.0\%]. Mean hospital length of stay was 1.77 days [95\% CI 1.21-2.24]. After a median follow-up of 6.6 months (1-24), the rate of recurrence was $1 \%$ [95\% CI 0.0-1.0\%].

Conclusion Minimally invasive eTEP is a safe and effective approach for ventral hernia repair, with low reported intraoperative complications and good outcomes.
\end{abstract}

Keywords Enhanced view totally extraperitoneal $\cdot$ eTEP $\cdot$ Minimally invasive surgery $\cdot$ Ventral hernia

\section{Introduction}

After the first description of laparoscopic ventral hernia repair by Leblanc et al. in 1993, the laparoscopic IPOM (intraperitoneal onlay mesh) technique rapidly became the established approach, as it led to faster recovery and fewer serious wound complications [1]. Subsequently, Agarwal et al. [2] improved the IPOM technique introducing defect

D. Aliseda

dalisedaj@unav.es

1 Department of General Surgery, Clinica Universidad de Navarra, University of Navarra, Av. Pío XII, 36, 31008 Pamplona, Spain

2 Institute of Health Research of Navarra (IdisNA), Pamplona, Spain closure. The defect closure performed in IPOM + reduced seroma rates [3] recurrence and bulging [4]. However, complications involving intraperitoneal mesh placement such as mesh adhesions, fistulation and migration became a problem. In addition, increased postoperative pain, in relation to mesh fixation [5], and higher reoperation rates [6] led to a significant drop in the number of laparoscopic IPOM repairs (from $33.8 \%$ in 2013 to $21.0 \%$ in 2019) [7]. As a result, there has recently been an increase in new minimally invasive approaches such as eTEP [7].

Described by Daes in 2012 for laparoscopic inguinal hernia, it was later adapted for ventral hernia repair, resulting in a technically challenging and demanding approach [8]. This technique positions the mesh in the retro-rectus space, without entering the abdominal cavity, to overcome the complications aforementioned and improve outcomes [7, 9]. Belyansky et al. published the first outcomes in 2018 from 
a multicenter study that showed the feasibility and safety of this ventral hernia repair technique [10].

Since then, various articles have been published reporting the outcomes of this approach for ventral hernia repair [11]. Despite this, studies with solid evidence supporting this technique are lacking. The objective of this study is to perform a systematic review of the literature and meta-analysis to summarize and ascertain the safety and short-term outcomes of eTEP for ventral hernia repair.

\section{Material and methods}

This systematic review was conducted in accordance with the latest updated Preferred Reporting Items for Systematic Reviews and Meta-Analyses (PRISMA) guidelines [12]. This review and the protocol were registered in the PROSPERO platform (CRD42021231029).

\section{Search strategy}

The PubMed/MEDLINE, EMBASE, Cochrane Library and Web of Science electronic databases were reviewed using the following search strategy combining keywords and using Boolean operators. The following key terms were used to identify relevant studies: "Rives Stoppa", "retro rectus" "retromuscular", "sublay repair", "enhanced view total extraperitoneal", "laparoscopic", "minimally invasive", "robotic", "hybrid", "ventral hernia" and "incisional hernia". All possible combinations of keywords were utilized. An additional search was conducted using bibliographic cross-referencing. The final search was performed in January 2021.

\section{Study selection}

The inclusion criteria were studies written in English or Spanish published between January 2005 and January 2021 that included adults over the age of 18 years diagnosed with ventral hernia (primary or incisional) according to the European Hernia Society (EHS) classification [13]. Patients undergoing concomitant procedures, or inguinal or parastomal hernia repair were excluded. The hernia repair had to be performed using a laparoscopic or robotic approach and using the enhanced view totally extraperitoneal technique as proposed by Belyanski et al. [10]. All the studies had to include a good description of technique and end points. Reviews, editorials, and case reports of $<5$ patients were excluded, as were manuscripts in which other minimally invasive hernia repair techniques were performed. When duplicates were detected, the largest series was selected.

All the articles retrieved were screened in duplicate. Two researchers (D.A and C.S-J) carried out the first blind screening in duplicate by reading titles and abstracts. The subsequent identification of articles to be included was performed by reading full texts, also in duplicate (D.A and C.S-J). In the process of identifying articles to be included, the rejected articles were correctly identified and the lack of fulfillment of the inclusion criteria was appropriately indicated (Supplementary material Table 1S). In the event of inclusion/exclusion discrepancies (both in screening and identification), a third investigator (P.M-C) was appointed as a referee, or any doubts were resolved by group consensus.

\section{Data extraction and quality assessment}

Data extraction was blinded and performed in duplicate (D.A and A.A) using a data extraction form specifically developed for the review, which was then cross-checked. From each study, at least the following items were extracted: title of the review, first author, year of publication, the authors' affiliation country, patient baseline characteristics, perioperative details, postoperative outcomes and followup. The methodological quality of the selected studies was assessed by D.A and an experienced external epidemiologist using Methodological Index for Non-randomized Studies (MINORS) criteria.

\section{Statistical analysis}

This meta-analysis was conducted with STATA version 16 (StataCorp, College Station, Texas 77845 USA). Continuous data were expressed as means or medians with standard derivations or ranges, as appropriate. Also, categorical variables were shown as numbers and percentages for descriptive purposes. The "metan" and "metaprop" programs were used to pool means and proportions with $95 \%$ confidence intervals, respectively. The STATA "metaprop" program allows the execution of meta-analyses of binomial data using the score statistic and the exact binomial method and includes the Freeman-Tukey double arcsine transformation of proportions by stabilizing between-study variance [13]. It is also a convenient method for dealing with proportions near the boundary ( 0 or 1$)$. Otherwise, studies would be excluded from the analysis resulting in a biased pooled estimate [13]. Furthermore, pooled proportions were derived from random effect models (because we assumed that the true effect estimated in each study varied due to differences in patient characteristics and assay types). Heterogeneity across studies was assessed with Cochran's $Q$ test, and based on the method reported by DerSimonian and Laird substantial significance was set when the $p$ value was $<0.10$. An $I$-square value of $<25 \%$ was defined to represent low heterogeneity, a value between 25 and $50 \%$ was defined as moderate heterogeneity, and a value $>50 \%$ was defined as high heterogeneity. 
A univariate meta-regression was also conducted with the "metareg" function. Analysis was done in a random effect-restricted maximum likelihood model with the Knapp-Hartung variance estimation accompanied by the use of a $t$ distribution in place of a normal distribution [14]. Univariate regression was considered for perioperative and short-term outcomes (dependent variable) with high heterogeneity $\left(I^{2}>50 \%\right)$ and corrected for conventional factors that could result in the variability in these outcomes (independent variables). Publication bias was visually explored using funnel plots, quantitively assessed using Egger's test and was considered to exist when $p<0.10$. In the case of articles only reporting the median, range and the size of the sample, to pool data, mean values and standard deviations were calculated using the formulas proposed by Hozo SP et al. [15]. All tests were two-sided with a significance level of 0.05 .

\section{Measures and endpoints}

Primary end points were short-term outcomes, in terms of:

\section{Perioperative characteristics}

These included intraoperative complications (using the Delphi study definition [16] and including conversion) conversion rate, length of hospital stay and operative time.

\section{Postoperative parameters}

These included wound complications (surgical site infection, seroma and hematoma), postoperative major morbidity (Clavien-Dindo III-IV [17]), reoperation, readmission and recurrence rate.

\section{Results}

\section{Search results}

A total of 8,462 studies were retrieved by the literature search. After removing duplicates, 268 articles were screened. No other studies were found via citation searching. A full-text review was performed on 47 reports from the electronic searches. Finally, 13 reports met the inclusion criteria for this systematic review (Table 1). The 2020 PRISMA flowchart with each step of the selection process is presented in Fig. 1.

\section{Study characteristics}

Within the 13 studies included, there were 12 observational studies (including one propensity-score study) and 1 interventional study [11]. Four studies were prospective (3 observational; 1 interventional) and nine were retrospective series (Table 1). Preoperative, perioperative and short term-postoperative characteristics of patients included in the studies are provided in Supplementary material Tables 2S, 3S and 4S.

A total of 918 patients from 12 institutions were included in this study. The study population was composed of 467 men and 451 women. Overall, patient mean age was 54.29 years $( \pm 1.28)$ with a mean BMI of $29.16 \mathrm{~kg} / \mathrm{m}^{2}$ $( \pm 0.58)$ with five studies $[10,18-21]$ presenting an obese population with a mean $\mathrm{BMI} \geq 30 \mathrm{~kg} / \mathrm{m}^{2}$ (Supplementary material Table 2S). Nine studies [11, 18-20, 22-26] reported the hernia etiology, with $45.7 \%$ primary ventral hernia. Nine
Table 1 Selected studies reporting the minimally invasive eTEP approach for ventral hernia repair

\begin{tabular}{lllllll}
\hline Author & Year & Country & No. of patients & Study design & Center & MINORS score \\
\hline Kumar N et al.[11] & 2020 & India & 46 & Prospective & Single & $19 / 24$ \\
Ngo P et al.[27] & 2020 & France & 112 & Prospective & Multi & $8 / 16$ \\
Salido S et al.[18] & 2020 & Spain & 40 & Prospective & Multi & $11 / 16$ \\
Prakhar G et al.[22] & 2020 & India & 171 & Retrospective & Single & $7 / 16$ \\
Mitura K et al. [19] & 2020 & Poland & 11 & Prospective & Single & $9 / 16$ \\
Sanna A et al. [23] & 2020 & Italy & 18 & Retrospective & Single & $9 / 16$ \\
Morrell ALG et al.[24] & 2020 & Brazil & 74 & Retrospective & Multi & $11 / 16$ \\
Kudsi OY et al.[20] & 2020 & USA & 82 & Retrospective & Single & $17 / 24$ \\
Köhler G et al.[25] & 2019 & Austria & 31 & Retrospective & Single & $11 / 16$ \\
Baig SJ et al.[28] & 2019 & India & 21 & Retrospective & Single & $7 / 16$ \\
Penchev D et al.[26] & 2019 & Bulgaria & 27 & Retrospective & Single & $17 / 24$ \\
Lu R et al.[21] & 2019 & USA & 206 & Retrospective & Single & $18 / 24$ \\
Belyansky I et al. [10] & 2017 & USA & 79 & Retrospective & Multi & $10 / 16$ \\
\hline
\end{tabular}

eTEP enhanced view totally extraperitoneal 
Fig. 1 Flowchart of study screening according to PRISMA guidelines
Identification of new studies via databases and registers
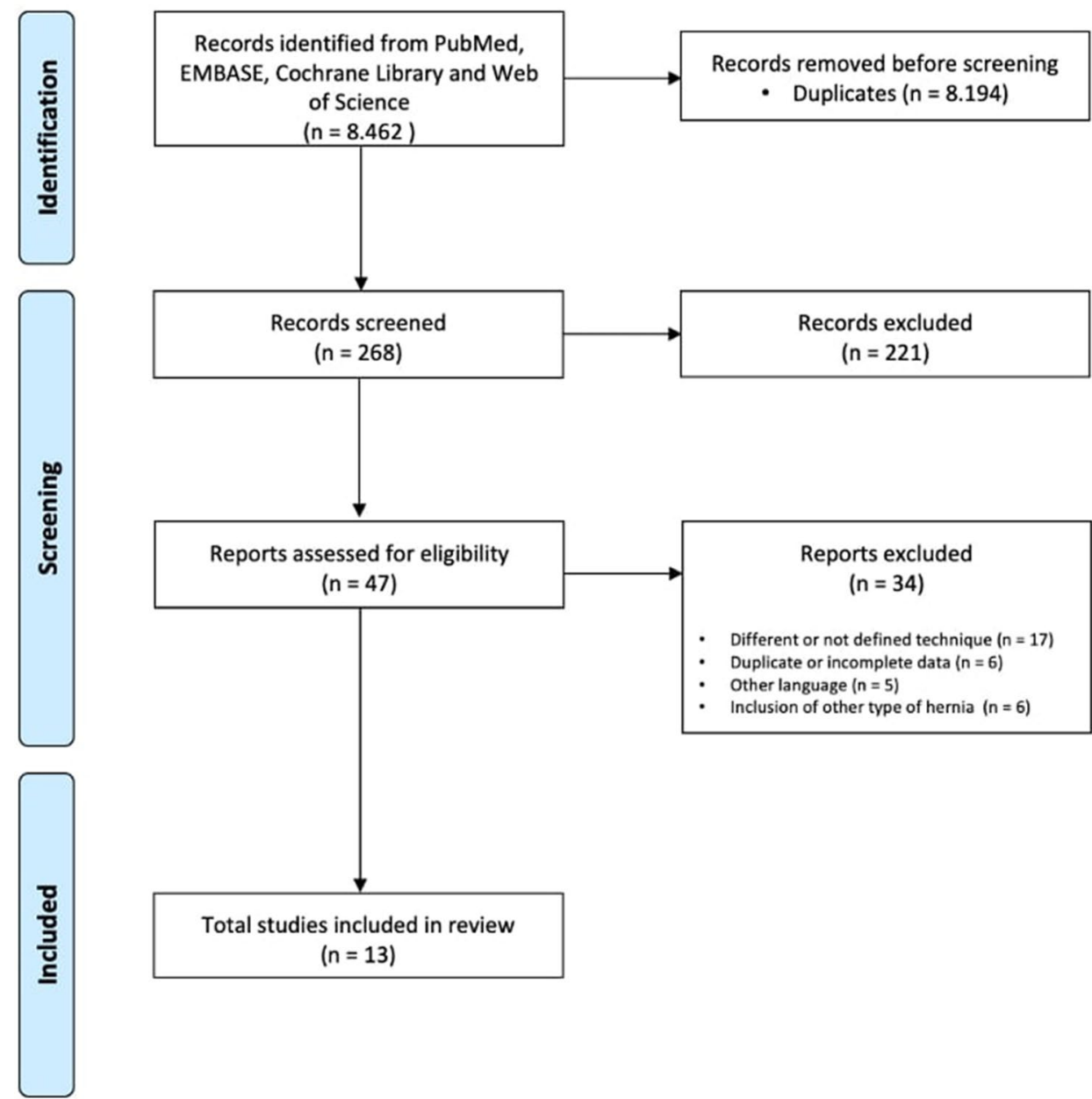

Total studies included in review $(n=13)$ studies $[11,18,19,22,23,25-28]$ provided data on hernia location according to the EHS classification [13] resulting in $96.2 \%$ of midline hernias and $3.8 \%$ of lateral hernias. Hernia size (width) was reported in eight studies [11, 18-21, 24, $25,28]$ and resulted in a mean size of $6.38 \mathrm{~cm}$ [5.12-7.63]. Information regarding covered mesh area is shown in Supplementary material Table $2 \mathrm{~S}$.

\section{Perioperative characteristics}

Laparoscopic eTEP was performed in ten studies, two studies reported totally robotic approach and one study showed the results of robotic and laparoscopic eTEP (Supplementary material Table $2 \mathrm{~S}$ ). The proportion of intraoperative complications was given in 10/13 studies (heterogeneity $p$ value $=0.03 ; I^{2}=50.91 \%$; with a proportion of $2 \%$ [95\% CI $0.0-4.0 \%]$ ) (Fig. 2). The conversion rate was reported in $10 / 13$ studies (heterogeneity $p$ value $=0.23 ; I^{2}=23.12 \%$; with a proportion of $1.0 \%$ [95\% CI 0.0-3.0\%]) (Fig. 3). Supplementary Table $2 \mathrm{~S}$ shows that the mean operative time was
148.89 min [95\% CI 129.45-168.34] and length of hospital stay was 1.77 days [95\% CI 1.21-2.24]. Within this review, in 117 cases $(12.7 \%$ ) (Supplementary material Table 3S) transversus abdominis release (TAR) was performed in association with RS mainly due to large defects or excessive tension on the posterior layer.

\section{Postoperative characteristics}

The rate of surgical site infection was reported in 11/13 studies (heterogeneity $p$ value $=0.63 ; I^{2}=0.0 \%$; with a rate of $0 \%$ [95\% CI $0.0-1.0 \%$ ]) (Fig. 4a). In terms of surgical site occurrence (SSO), seroma and hematoma were evaluated. Twelve reports assessed seroma rates (heterogeneity $p$ value $=0.000 ; I^{2}=63.23 \%$; with a rate of 5\% [95\% CI 2.0-8.0\%]) (Fig. 4b) and 12/13 studies reported hematoma rates (heterogeneity $p$ value $=0.23$; $I^{2}=21.54 \%$; with a rate of $1 \%$ [95\% CI $\left.0.0-2.0 \%\right]$ ) (Fig. 4c). The rate of major complications (Clavien-Dindo 
Fig. 2 Forest plot of intraoperative complication rate

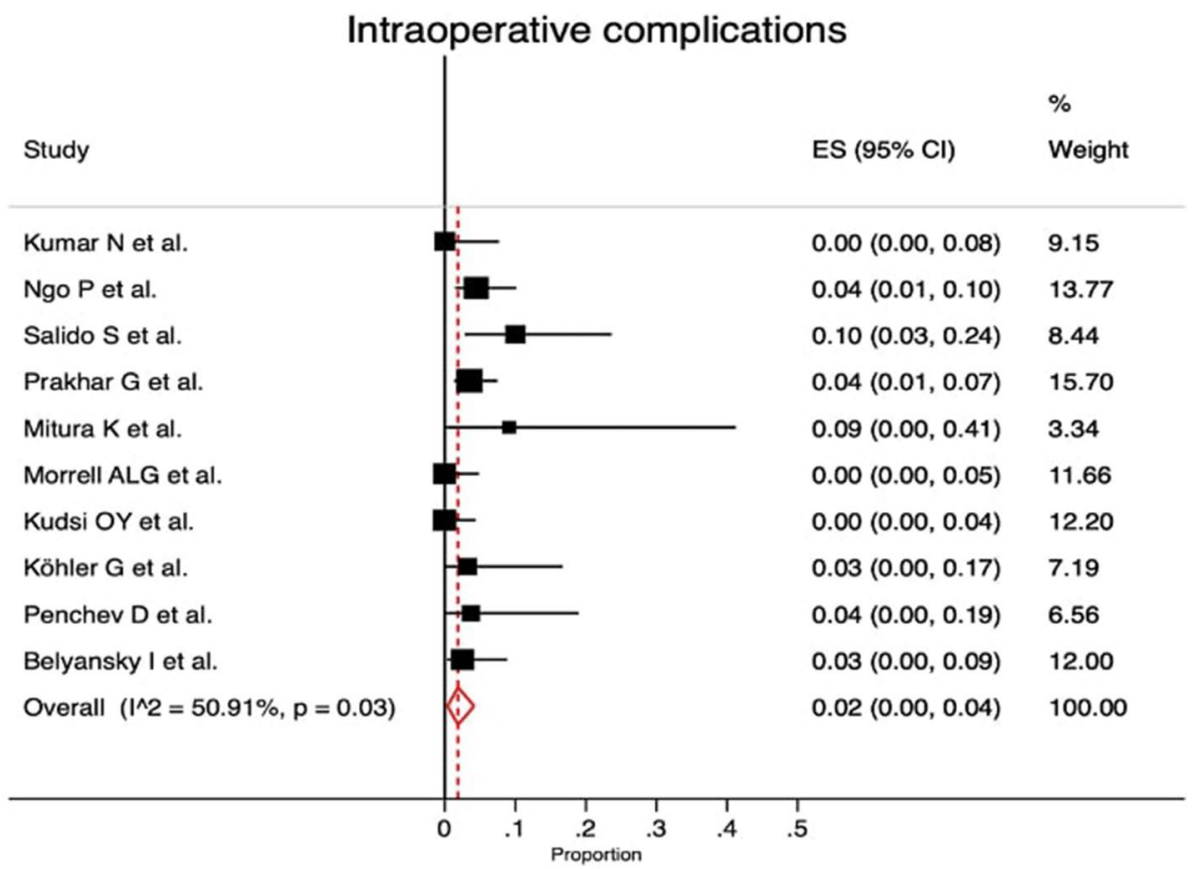

Fig. 3 Forest plot of conversion rate

III-IV) was calculated in $12 / 13$ studies (heterogeneity $p$ value $=0.42 ; I^{2}=2.91 \%$; with a rate of $1 \%[95 \% \mathrm{CI}$ $0.0-3.0 \%]$ ) (Fig. 4d). The reoperation rate was reported in $13 / 13$ (heterogeneity $p$ value $=0.41 ; I^{2}=3.40 \%$; with a rate of $1 \%$ [95\% CI 0.0-2.0\%]) (Fig. 4e). The readmission rate was provided in $13 / 13$ (heterogeneity $p$ value $=0.20$; $I^{2}=23.98 \%$; with a rate of $1 \%$ [95\% CI $\left.0.0-3.0 \%\right]$ ) (Fig. 4f).

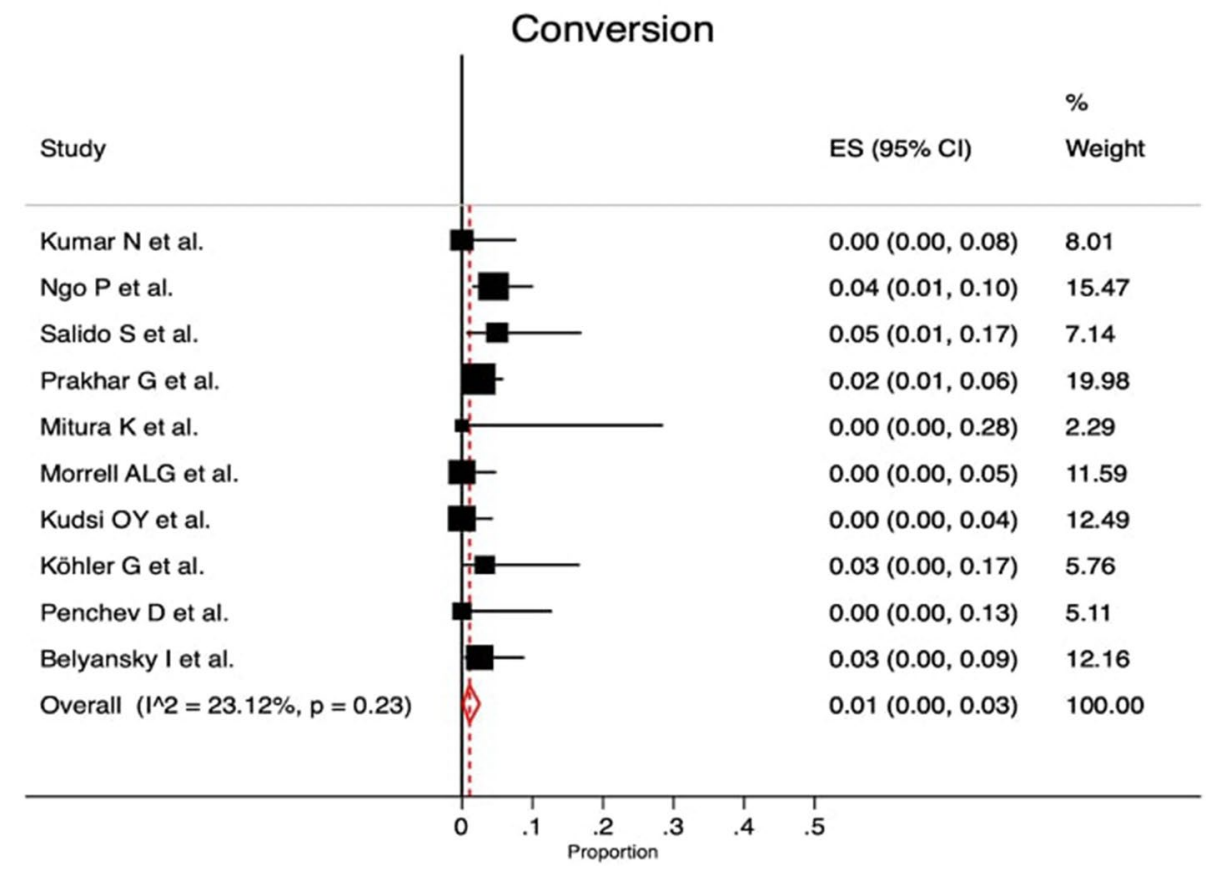

Follow-up ranged from 1 to 24 months with a median of 6.6 months (Supplementary material Table 4S). Twelve studies reported recurrence (heterogeneity $p$ value $=0.73$; $I^{2}=0.0 \%$; with a rate of $1 \%$ [95\% CI 0.0-1.0\%]) (Fig. 5). 
(a) Surgical site infection (SSI)

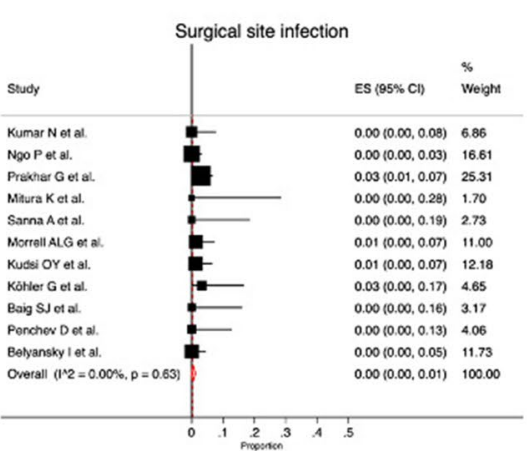

(d) Major complications

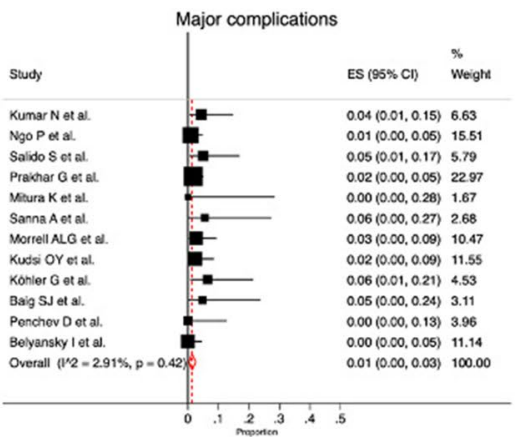

(b) Seroma

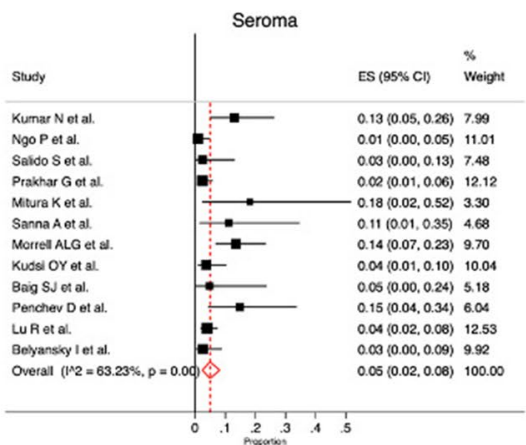

(e) Reoperation

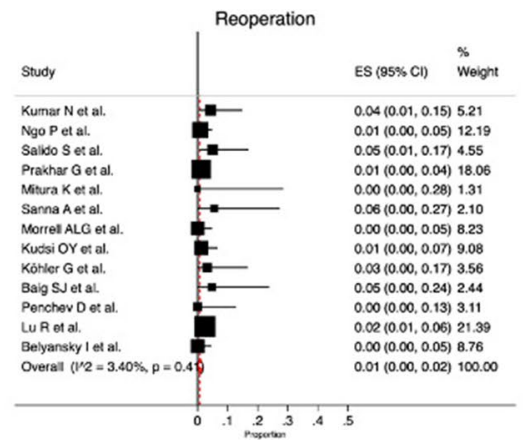

(c) Hematoma

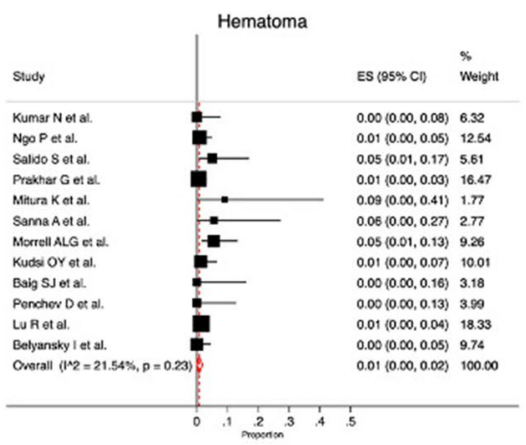

(f) Readmission

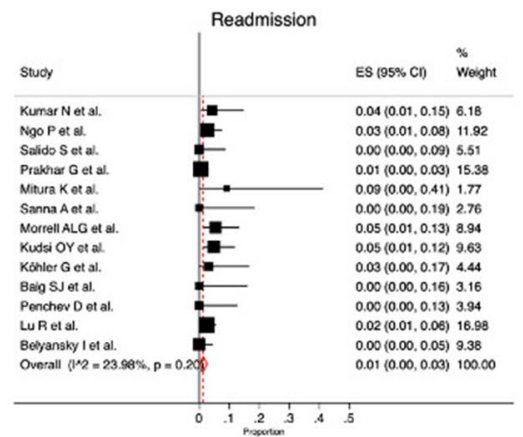

Fig. 4 Meta-analysis of short-term outcomes

Fig. 5 Forest plot of recurrence rate

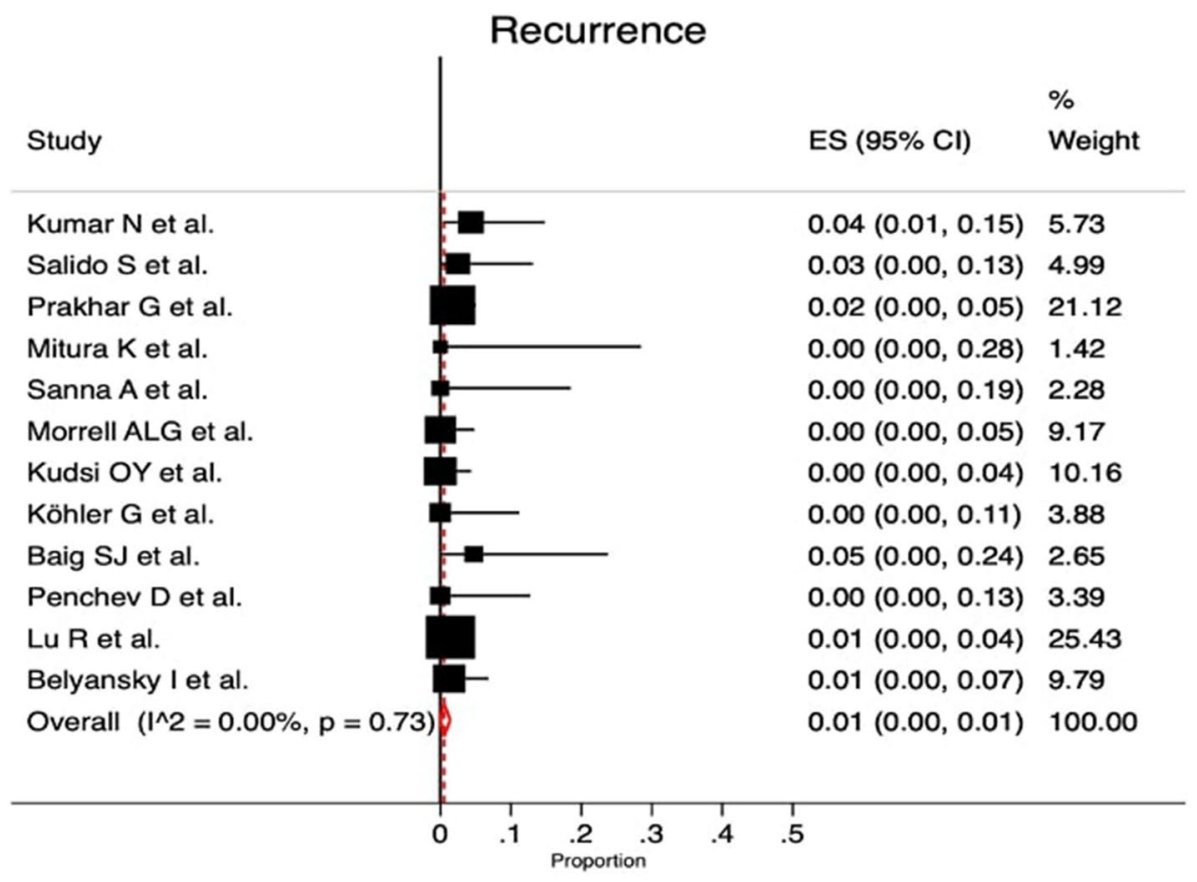




\section{Meta-regression analysis}

A meta-regression was performed to explore sources of heterogeneity in intraoperative complication and seroma rates including: minimally invasive (MI) approach (laparoscopic vs robotic), study size ( $<50$ vs. $\geq 50$ patients), age (mean age $<55$ vs. $\geq 55$ years) and obesity (mean $\mathrm{BMI}<30$ vs. $\geq 30 \mathrm{~kg} / \mathrm{m}^{2}$ ). Meta-regression analyses determined that MI approach did not show a statistical trend for intraoperative complications rate $(\beta=-0.0387$; SE: $0.0910 ; p=0.682)$ and seroma rate $(\beta=0.0407 ; \mathrm{SE}=0.0879 ; p=0.653)$. Moreover, study size did not show a statistical trend for intraoperative complication rate $(\beta=-0.0387$; SE: $0.0910 ; p=0.682)$ and seroma rate $(\beta=0.0407 ; \mathrm{SE}=0.0879 ; p=0.653)$. Neither age $(\beta=-0.0147$; $\mathrm{SE}=0.0720 ; p=0.842)$ nor BMI $(\mathrm{kg} /$ $\left.\mathrm{m}^{2}\right)(\beta=-0.0214 ; \mathrm{SE}=0.0670 ; p=0.755)$ was significantly identified as confounding factors in seroma rates. The same results were found in terms of intraoperative complications rates where age $(\beta=0.0140 ; \mathrm{SE}=0.0774 ; p=0.861)$ and BMI $\left(\mathrm{kg} / \mathrm{m}^{2}\right)(\beta=0.0050 ; \mathrm{SE}=0.0826 ; p=0.953)$ were not significantly associated as confounding factors.

\section{Publication bias analysis}

All the studies were independently assessed for bias in each variable using funnel plots and Egger's test (Supplementary material Figure S1-S9B). Publication bias was found for seroma rates (Supplementary material Figure S4B).

\section{Methodological quality of studies}

The comparative and the non-comparative studies achieved a median MINORS score of 17.5/24 (range 17-19) and 9/16 (range 7-11), respectively. The MINORS score evaluation for each study is shown in Supplementary material (Table 5S).

\section{Discussion}

This is, to our knowledge, the first systematic review and meta-analysis that provides a comprehensive overview of the available evidence regarding the feasibility, safety and short-term outcomes of eTEP for ventral hernia repair.

\section{Perioperative characteristics}

The eTEP is a set of maneuvers and operative strategies which was developed to overcome the limits of the TEP approach for inguinal hernia and was later adapted for the surgical treatment of medial and lateral ventral hernias [8]. These maneuvers are able to broaden the extraperitoneal space in a minimally invasive approach. The eTEP is a challenging technique that requires a thorough knowledge of the anatomy of the extraperitoneal space and where advanced laparoscopic skills are needed to perform some maneuvers successfully [29]. In addition, there are multiple approaches and technical aspects to consider when approaching ventral hernia repair with eTEP [29].

As a consequence of the difficulty of this technique as well as its recent implementation, several articles reported a longer operative time for the eTEP approach compared with other minimally invasive ventral hernia repair techniques [30]. However, once the learning curve is completed, a decrease in operative times and adverse outcomes is observed [31].

Despite the complexity of this technique, according to this study eTEP seems to be safe in terms of intraoperative events. The rate of intraoperative complications and conversion in this meta-analysis was $2.0 \%$ and $1.0 \%$, respectively. These results appear to be similar compared with laparoscopic IPOM and seems to improve those derived from sublay OVHR [32]. Not entering the abdominal cavity is a favorable characteristic of this technique, avoiding adhesiolyisis and its associated complications [33, 34]. In fact, eTEP seems to improve one of the problems associated with IPOM and IPOM +, avoiding mesh fixation with tacks or transabdominal sutures and thus decreasing postoperative pain [5]. However, a note of caution must be sounded, since the risk of bowel injury is still present due to thermal injury behind the posterior layer and also during the crossover maneuver, especially in patients who have had previous abdominal surgery [18].

Recent studies have shown a reduction of pain and a better functional recovery when comparing eTEP with IPOM+ $[11,35]$. This pain reduction due to unnecessary mesh fixation may be one of the reasons for the differences in length of hospital stay between IPOM and eTEP. In this study, hospital stay was 1.77 days, which appears to be in line with other transabdominal extra- and intraperitoneal minimally invasive ventral hernia repair techniques [20,35] and seems to improve over OVHR [36], including open sublay technique[32].

In addition to these advantages, the vast majority of studies reporting on eTEP approach used uncoated polypropylene meshes. These meshes are affordable, thus decreasing the costs of the procedure compared to the IPOM, where much more expensive meshes with an adhesion barrier are commonly used [37].

Another interesting aspect to consider is the irruption of robotic surgery for eTEP. The introduction of robotic approach has allowed to optimize some maneuvers and subsequently operate more complex patients [21]. The threedimensional view and freedom of movement that the robotic approach introduces may help in those technically difficult maneuvers and in intracorporeal suturing. These benefits 
lead to shorter lengths of stay with similar morbidity as compared to laparoscopic approaches as Warren JA et al. have reported [38].

\section{Postoperative characteristics}

During the dissection of the retromuscular plane, a large space is created with the consequent risk of seroma and hematoma formation. In the literature, the rates of seroma and hematoma formation of LVHR differ significantly between studies with studies reporting a significantly higher seroma rate of IPOM + compared with eTEP and vice versa $[11,35]$. Anyway, more studies are needed to clarify this matter.

There are several specific complications related to the eTEP approach. Such complications as injury to the linea alba, retromuscular hematoma or injury to the neurovascular bundle could - theoretically — increase morbidity and reoperation rates especially at the beginning of the learning curve [10]. Recently, Henriksen et al. [6] showed a rate of reoperation of $5.0 \%$ and $2.7 \%$ of OVHR and laparoscopic IPOM, respectively. Furthermore, a large propensity scorematched comparison of almost 10,000 patients showed postoperative surgical complication rates of a $3.4 \%$ and $10.5 \%$ after laparoscopic IPOM and sublay OVHR, respectively [32]. Therefore, based on this meta-analysis, in the hands of well-trained hernia surgeons, the eTEP approach seems to be safe in terms of major complications and reoperation. However, we firmly believe that it is of utmost importance to emphasize the need for adequate preparation before engaging in any of these complex repairs. As the pioneers of the eTEP approach point out, training is of vital importance to perform safe surgery with good results [29].

A recent meta-analysis of 51 articles showed that retromuscular mesh repair is associated with a lower recurrence rate [39]. However, dissecting the retromuscular plane requires the correct restoration of this space prior to the placement of the mesh. During eTEP, the incomplete closure of the posterior layer due to technical demanding maneuvers or the breakdown of the posterior layer because of increased tension are specific limitations that may increase recurrence and complications [11]. For this reason, after appropriate training, it is recommended to start using this technique by repairing small midline hernias associated with diastasis recti [29]

The rate of recurrence after one-year follow-up of laparoscopic IPOM and sublay OVHR according to Kökerling et al. [32] was $4.2 \%$ and $4.1 \%$, respectively. Based on this meta-analysis, eTEP seems to be effective in the repair of ventral hernia with a low recurrence rate. However, the follow-up period of this study is short, so that to correctly assess recurrence, studies with a larger follow-up would be needed to confirm this result.

\section{Limitations}

The limitations of this systematic review and meta-analysis are related to the small number of published studies on this technique as well as the heterogeneity of the studies, the sample size and the observational design of most studies included. Another aspect to bear in mind is the short follow-up to evaluate recurrence and the difference in surgical experience, between studies, with the MI and eTEP technique. Furthermore, it should be taken into account that the surgeries included in this study were performed by expert surgeons in the field of minimally invasive abdominal wall surgery and some of them are even pioneers in the eTEP approach for ventral hernia. For this reason, the good results of this study should be considered in a balanced way and these aspects should be taken into account when assessing its reproducibility. Despite its limitations, the present study offers an objective summary of the evidence of MI eTEP approach for ventral hernia repair that could be useful in guiding surgical decisions.

\section{Conclusion}

This systematic review and meta-analysis summarize the perioperative and postoperative short-term outcomes of minimally invasive eTEP. The outcomes observed in this meta-analysis suggest that, in the hands of well-trained hernia surgeons, minimally invasive eTEP is a safe and effective approach for ventral hernia repair. Despite this, large randomized clinical trials are needed to evaluate the short- and long-term outcomes of this new technique.

Supplementary Information The online version contains supplementary material available at https://doi.org/10.1007/s10029-021-02557-8.

Author contributions DA: conceptualization, methodology, validation, formal analysis, data curation, writing — original draft, writing — review and editing, visualization. PM-C: validation, writing - review and editing. GZ: writing - review and editing. CS-J: data curation-validation, writing — review and editing. JL: writing — review and editing. NB: writing - review and editing. AA: data curation-validation, writingreview and editing. FR: writing-review and editing.

Funding Open Access funding provided thanks to the CRUE-CSIC agreement with Springer Nature.

\section{Declarations}

Conflict of interest Drs. Daniel Aliseda, Carlos Sanchez-Justicia, Gabriel Zozaya, Juan Luján, Ana Almeida, Nuria Blanco, Pablo MartíCruchaga and Fernando Rotellar have no conflicts of interest or financial ties to disclose.

Ethical approval This article does not contain any studies with human participants or animal performed by any of the authors. 
Human and animal rights This article does not contain any studies directly involving human participants, as it is a review of data already collected and publicated.

Informed consent For this type of study, formal consent is not required.

Open Access This article is licensed under a Creative Commons Attribution 4.0 International License, which permits use, sharing, adaptation, distribution and reproduction in any medium or format, as long as you give appropriate credit to the original author(s) and the source, provide a link to the Creative Commons licence, and indicate if changes were made. The images or other third party material in this article are included in the article's Creative Commons licence, unless indicated otherwise in a credit line to the material. If material is not included in the article's Creative Commons licence and your intended use is not permitted by statutory regulation or exceeds the permitted use, you will need to obtain permission directly from the copyright holder. To view a copy of this licence, visit http://creativecommons.org/licenses/by/4.0/.

\section{References}

1. LeBlanc KA, Booth WV (1993) Laparoscopic repair of incisional abdominal hernias using expanded polytetrafluoroethylene: preliminary findings. Surg Laparosc Endosc 3(1):39-41

2. Agarwal BB, Agarwal S, Mahajan KC (2009) Laparoscopic ventral hernia repair: innovative anatomical closure, mesh insertion without $10-\mathrm{mm}$ transmyofascial port, and atraumatic mesh fixation: a preliminary experience of a new technique. Surg Endosc 23:900-905. https://doi.org/10.1007/s00464-008-0159-7

3. Suwa K, Okamoto T, Yanaga K (2016) Closure versus non-closure of fascial defects in laparoscopic ventral and incisional hernia repairs: a review of the literature. Surg Today 46:764-773. https:// doi.org/10.1007/s00595-015-1219-y

4. Mitura K, Skolimowska-Rzewuska M, Garnysz K (2017) Outcomes of bridging versus mesh augmentation in laparoscopic repair of small and medium midline ventral hernias. Surg Endosc 31:382-388. https://doi.org/10.1007/s00464-016-4984-9

5. Brill JB, Turner PL (2011) Long-term outcomes with transfascial sutures versus tacks in laparoscopic ventral hernia repair: a review. Am Surg 77:458-465. https://doi.org/10.1177/000313481107700 423

6. Henriksen NA, Jorgensen LN, Friis-Andersen H et al (2021) Open versus laparoscopic umbilical and epigastric hernia repair: nationwide data on short- and long-term outcomes. Surg Endosc. https:// doi.org/10.1007/s00464-021-08312-5

7. Köckerling F, Hoffmann H, Mayer F et al (2020) What are the trends in incisional hernia repair? Real-world data over 10 years from the Herniamed registry. Hernia. https://doi.org/10.1007/ s10029-020-02319-y

8. Daes J (2012) The enhanced view-totally extraperitoneal technique for repair of inguinal hernia. Surg Endosc 26:1187-1189. https://doi.org/10.1007/s00464-011-1993-6

9. Gokcal F, Morrison S, Kudsi OY (2019) Short-term comparison between preperitoneal and intraperitoneal onlay mesh placement in robotic ventral hernia repair. Hernia 23:957-967. https://doi. org/10.1007/s10029-019-01946-4

10. Belyansky I, Daes J, Radu VG et al (2018) A novel approach using the enhanced-view totally extraperitoneal (eTEP) technique for laparoscopic retromuscular hernia repair. Surg Endosc 32:15251532. https://doi.org/10.1007/s00464-017-5840-2
11. Kumar N, Palanisamy NV, Parthasarathi R et al (2020) A comparative prospective study of short-term outcomes of extended view totally extraperitoneal (e-TEP) repair versus laparoscopic intraperitoneal on lay mesh (IPOM) plus repair for ventral hernia. Surg Endosc. https://doi.org/10.1007/s00464-020-07990-x

12. Pagew MJ, McKenzie JE, Bossuyt PM et al (2020) The PRISMA 2020 statement: an updated guideline for reporting systematic reviews. BMJ. https://doi.org/10.1136/bmj.n71

13. Muysoms FE, Miserez M, Berrevoet F et al (2009) Classification of primary and incisional abdominal wall hernias. Hernia 13:407-414. https://doi.org/10.1007/s10029-009-0518-x

14. Harbord RM, Higgins JPT (2008) Meta-regression in stata. Stata J 8:493-519. https://doi.org/10.1177/1536867x0800800403

15. Hozo SP, Djulbegovic B, Hozo I (2005) Estimating the mean and variance from the median, range, and the size of a sample. Bmc Med Res Methodol 5:13. https://doi.org/10.1186/1471-2288-5-13

16. Rosenthal R, Hoffmann H, Clavien P-A et al (2015) Definition and classification of intraoperative complications (CLASSIC): delphi study and pilot evaluation. World J Surg 39:1663-1671. https:// doi.org/10.1007/s00268-015-3003-y

17. Dindo D, Demartines N, Clavien P-A (2004) Classification of surgical complications. Ann Surg 240:205-213. https://doi.org/ 10.1097/01.sla.0000133083.54934.ae

18. Fernandez SS, Vilarrasa MF, Silla IO et al (2020) Vía totalmente extraperitoneal extendida (eTEP) para la corrección de hernias ventrales: resultados preliminares. Cir Esp 98:260-266. https:// doi.org/10.1016/j.ciresp.2020.01.006

19. Mitura K, Rzewuska A, Skolimowska-Rzewuska M et al (2020) Laparoscopic enhanced-view totally extraperitoneal Rives-Stoppa repair (eTEP-RS) for ventral and incisional hernias-early operative outcomes and technical remarks on a novel retromuscular approach. Videosurgery Miniinv 15:533-545. https://doi.org/10. 5114/wiitm.2020.99371

20. Kudsi OY, Chang K, Bou-Ayash N, Gokcal F (2020) Transabdominal (TA) versus totally extraperitoneal (TEP) robotic retromuscular ventral hernia repair: a propensity score matching analysis. Surg Endosc 34:3550-3559. https://doi.org/10.1007/ s00464-020-07574-9

21. Lu R, Addo A, Ewart Z et al (2020) Comparative review of outcomes: laparoscopic and robotic enhanced-view totally extraperitoneal (eTEP) access retrorectus repairs. Surg Endosc 34:35973605. https://doi.org/10.1007/s00464-019-07132-y

22. Prakhar G, Parthasarathi R, Cumar B et al (2020) Extended view: totally extra peritoneal (e-TEP) approach for ventral and incisional hernia-early results from a single center. Surg Endosc. https:// doi.org/10.1007/s00464-020-07595-4

23. Sanna A, Felicioni L, Cecconi C, Cola R (2020) Retromuscular mesh repair using extended totally extraperitoneal repair minimal access: early outcomes of an evolving technique - a single institution experience. J Laparoendosc Adv S 30:246-250. https://doi. org/10.1089/lap.2019.0661

24. Morrell ALG, Morrell AC, Cavazzola LT et al (2020) Robotic assisted eTEP ventral hernia repair: Brazilian early experience. Hernia. https://doi.org/10.1007/s10029-020-02233-3

25. Köhler G, Kaltenböck R, Pfandner R et al (2020) Precostal top-down extended totally extraperitoneal ventral hernia plasty (eTEP): simplification of a complex technical approach. Hernia 24:527-535. https://doi.org/10.1007/s10029-019-02076-7

26. Penchev D, Kotashev G, Mutafchiyski V (2019) Endoscopic enhanced-view totally extraperitoneal retromuscular approach for ventral hernia repair. Surg Endosc 33:3749-3756. https://doi. org/10.1007/s00464-019-06669-2

27. Ngo P, Cossa J-P, Largenton C et al (2020) Ventral hernia repair by totally extraperitoneal approach (VTEP): technique description 
and feasibility study. Surg Endosc. https://doi.org/10.1007/ s00464-020-07519-2

28. Baig SJ, Priya P (2019) Extended totally extraperitoneal repair (eTEP) for ventral hernias: short-term results from a single centre. J Minim Access Surg 15(3):198-203. https://doi.org/10.4103/ jmas.jmas_29_18

29. Daes J, Belyansky I (2021) Anatomical considerations and tips for laparoscopic and robotic-assisted enhanced-view totally extraperitoneal rives-stoppa repair for midline hernia. J Am Coll Surg 233:e1-e11. https://doi.org/10.1016/j.jamcollsurg.2021.05.007

30. Kudsi OY, Chang K, Bou-Ayash N, Gokcal F (2020) A comparison of robotic mesh repair techniques for primary uncomplicated midline ventral hernias and analysis of risk factors associated with postoperative complications. Hernia. https://doi.org/10.1007/ s10029-020-02199-2

31. Kudsi OY, Bou-Ayash N, Gokcal F et al (2020) Learning curve of robotic rives-stoppa ventral hernia repair: a cumulative sum analysis. J Laparoendosc Adv S. https://doi.org/10.1089/lap.2020. 0624

32. Köckerling F, Simon T, Adolf D et al (2019) Laparoscopic IPOM versus open sublay technique for elective incisional hernia repair: a registry-based, propensity score-matched comparison of 9907 patients. Surg Endosc 33:3361-3369. https://doi.org/10.1007/ s00464-018-06629-2

33. Mavros MN, Velmahos GC, Larentzakis A, Yeh DD, Fagenholz P, de Moya M, King DR, Lee J, Kaafarani HM (2014) Opening Pandora's box: understanding the nature, patterns, and 30-day outcomes of intraoperative adverse events. J Surg Res 186(2):497. https://doi.org/10.1016/j.jss.2013.11.088
34. Yang GPC (2017) From intraperitoneal onlay mesh repair to preperitoneal onlay mesh repair. Asian J Endosc Surg 10:119-127. https://doi.org/10.1111/ases.12388

35. Luque JB, Rosado JCG, Luque AB et al (2021) Endoscopic retromuscular technique (eTEP) vs conventional laparoscopic ventral or incisional hernia repair with defect closure (IPOM +) for midline hernias. A case-control study. Hernia. https://doi.org/10. 1007/s10029-021-02373-0

36. Zolin SJ, Tastaldi L, Alkhatib H et al (2020) Open retromuscular versus laparoscopic ventral hernia repair for medium-sized defects: where is the value? Hernia 24:759-770. https://doi.org/ 10.1007/s10029-019-02114-4

37. Prasad P, Tantia O, Patle NM et al (2011) Laparoscopic ventral hernia repair: a comparative study of transabdominal preperitoneal versus intraperitoneal onlay mesh repair. J Laparoendosc Adv S 21:477-483. https://doi.org/10.1089/lap.2010.0572

38. Warren JA, Cobb WS, Ewing JA, Carbonell AM (2017) Standard laparoscopic versus robotic retromuscular ventral hernia repair. Surg Endosc 31:324-332. https://doi.org/10.1007/ s00464-016-4975-x

39. Sosin M, Nahabedian MY, Bhanot P (2018) The perfect plane. Plast Reconstr Surg 142:107S-116S. https://doi.org/10.1097/prs. 0000000000004864

Publisher's Note Springer Nature remains neutral with regard to jurisdictional claims in published maps and institutional affiliations. 